\title{
CHARACTERISTIC-BASED VOLUME PENALIZATION METHOD FOR COMPRESSIBLE FLOW SIMULATIONS ON UNSTRUCTURED MESHES
}

\author{
ILYA V. ABALAKIN, TATIANA K. KOZUBSKAYA, \\ OLEG V. VASILYEV AND NATALIA S. ZHDANOVA \\ Keldysh Institute of Applied Mathematics, \\ 4A, Miusskaya Sq., Moscow, 125047, Russia \\ nat.zhdanova@gmail.com, http://caa.imamod.ru/
}

Key words: Unstructured Mesh, Immersed Boundary Method, Edge-Based Scheme, Brinkman Penalization, Characteristic-Based Volume Penalization, Compressible Flow

\begin{abstract}
The Characteristic-Based Volume Penalization (CBVP) method for numerical simulations of compressible flow over solid obstacles on unstructured meshes is presented. The approach belongs to the class of immersed boundary methods and is not relying on body-fitted meshes. Characteristic penalization terms, added to the compressible Navier-Stokes equations, are used to impose Dirichlet and Neumann boundary conditions on solid-fluid interface with an a priori defined accuracy. The details of numerical implementation, utilizing hybrid finitevolume method with high order edge-based reconstruction schemes in the flow region and loworder finite-difference approximation inside of the obstacle, are discussed. The developed algorithm provides the ability to perform calculations on grids of arbitrary type, including fully unstructured meshes. The efficiency of the characteristic based volume penalization method and its numerical implementation is demonstrated for shock wave reflection, acoustic pulse reflection and Couette flow problems. The results of CBVP simulations are compared with the numerical solutions of the same problems using Brinkman volume penalization method.
\end{abstract}

\section{INTRODUCTION}

Traditionally aerodynamic flow simulations are based on body-fitted meshes with solid-fluid interface defined by mesh nodes, where the boundary conditions are explicitly imposed. If an obstacle has a complicated geometry the construction of such meshes can become computationally expensive problem. The use of immersed boundary approach to model boundary conditions for such problems provides an attractive alternative because its implementation does not require the use of body-conformal computational meshes.

The volume penalization methods represent a separate sub-class of continuous immersed boundary methods, where the presence of an obstacle is mimicked by adding penalization terms to the system of governing equations. In Brinkman volume penalization (BP) method the obstacle is modelled as a porous medium with low permeability [1]. The formulation utilizes Brinkman-type penalization terms applied to the momentum and energy equations inside of the obstacle. One of advantages of the BP method is the ability to control the error through the penalization parameter with the proven convergence of the solution of the penalized Navier- 
Stokes equations to the exact solution in the limit when the penalization parameter tends to zero [2].

The BP method was first proposed in Ref. [1] and was successfully used for incompressible flows simulations $[3,4,5,6]$. The consistent extension of the Brinkman volume penalization method was proposed in Refs. [7, 8], where for correct reflection and transmission of sound waves through a porous medium in addition to Brinkman penalization of momentum and energy equations, the continuity equation is also modified inside the obstacle so that the penalized porous region acts as a high impedance medium.

The Brinkman penalization method, despite its ability to rigorously control the error of the solution, is limited to problems with Dirichlet boundary conditions. This drawback was recently overcome in the Characteristic-Based Volume Penalization (CBVP) method [9] that exploits the hyperbolicity of characteristic-based forcing terms to impose general homogeneous and inhomogeneous Neumann and Robin boundary conditions. The CBVP method was successfully applied to numerical simulation of the diffusion problem, an acoustic pulse reflection, and laminar flow around a two-dimensional cylinder. The application of the CBVP method was demonstrated for the Adaptive Wavelet Collocation Method [10], which provides active control of the solution error and the required local mesh resolution through dynamic structured grid adaptation.

In the present paper, the CBVP method is further refined and demonstrated for numerical simulations of viscous compressible flows over rigid obstacles using hybrid finite-volume/finite difference method on unstructured computational meshes with high-order edge-based reconstruction (EBR) applied in fluid and soli is verified by numerica including acoustic pulse and shock wave reflection problems a

2 CHARCTERISTIC-BASED VOLUME PENALIZATION METHOD

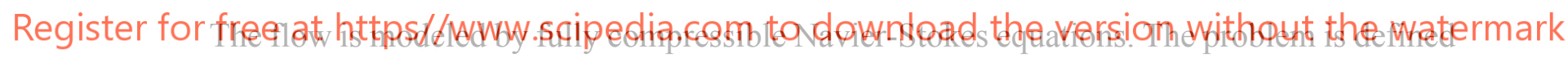

in the domain $\Omega$ containing the obstacle $\Omega_{B}$ surrounded by the fluid, occupying the domain

$\Omega_{E}$. The boundary between $\Omega_{B}$ and $\Omega_{E}$ is denoted by $\partial \Omega_{B}$ and the whole domain is $\Omega=\Omega_{E} \cup \bar{\Omega}_{B}$.

For the test problems, considered in this paper, the no-slip $\mathbf{u}_{\partial \Omega_{B}}=\mathbf{u}_{w}$ and adiabatic boundary conditions $\left.(\nabla \varepsilon, \mathbf{n})\right|_{\partial \Omega_{B}}=0$ are respectively imposed on velocity and internal energy, where $\mathbf{n}$ is inward-pointing normal at the boundary $\partial \Omega_{B}$ and $\mathbf{u}_{w}$ is the velocity of the moving obstacle.

The CBVP method [9] with no-slip boundary conditions for the velocity can be written in the following form:

$$
\frac{\partial u_{i}}{\partial t}=(1-\chi) R H S_{u_{i}}-\underbrace{\frac{\chi}{\eta_{b}}\left(u_{i}-u_{w, i}\right)}_{\text {penally term }}+\underbrace{\chi v_{n} \Delta u_{i}}_{\text {diffusion term }},
$$

where $\eta_{b} \ll 1$ is the penalization parameter and $\chi(\mathbf{x}, t)$ is the mask function, which is unity 
inside and zero outside of the obstacle.

The penalization parameter $\eta_{b}$ controls the error on the solution by decreasing the timescale of the forcing term. To ensure the smoothness of the solution, a diffusion term $v_{n} \Delta u_{i}$ is added to Eq. (1), where $v_{n}$ is the numerical diffusion proportional to the size of the mesh cell. The term $(1-\chi) R H S_{u_{i}}$ in Eq. (1) is used to remove physical terms from within the obstacle in order to prevent secondary effects of coupled systems of equations from contaminating the required boundary conditions. This omission of the RHS together with introduction of numerical diffusion results in consistent control over the penalization error and the resolution inside of the obstacle, regardless of the external flow physics.

The Neuman boundary conditions in the CBVP method are imposed through the characteristic forcing upon the normal derivative and have the following form:

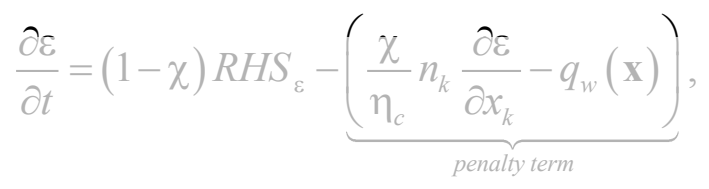

where $\eta_{c} \ll 1$ is the penalization parameter, which, in general, is different from the Brinkman penalization parameter $\eta_{b}$ used in Eq. (2). Note that for the sake of generality Eq. (2) is written for inhomogeneous Neumann boundary conditions. In the case of adiabatic boundary conditions for the internal energy (or tempdrature) the penalization target is set to zerd: $q_{w}(\mathbf{x})=0$.

The penalization teri the normal to the boundiry

from the scalar distance function: $\mathbf{n}(\mathbf{x})=\nabla \delta(\mathbb{x}), \mathbf{x} \in \Omega_{B}$ where $\delta$ is the minimal distance to

Register for freedrattps//www.scipedia.com to download the version without the watermark

From a mathematical point of view, the meaning of Eq. (2) is to propagate the solution from the surface along inward-pointing characteristics that enforce the desired value of derivative at the boundary $\partial \Omega_{B}$. Since the penalization timescale is controlled through $\eta_{c}$, selecting $\eta_{c} \ll 1$ causes the Eq. (2) to becomes quasi-steady within the domain $\Omega_{B}$ on the normalized problem timescale $t_{H}=L / U_{0}$ ( $L$ - typical obstacle size, $U_{0}$ - typical flow velocity). For dimensionless variables, denoted by "tilde", the Eq. (2) in the obstacle domain can be rewritten as

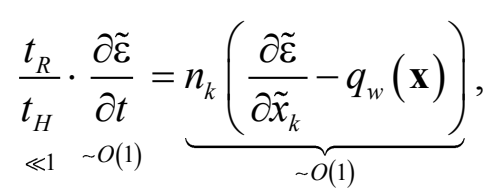

where $t_{R}=\eta_{c} L$ is the relaxation time scale. Since for moderate flow velocities $t_{R} / t_{H}=U_{0} \eta_{c} \ll 1$, then it follows from Eq. (3) that the boundary condition is satisfied on the time scale $O\left(t_{R}\right)$ with an asymptotic error of order $O\left(U_{0} \eta_{c}\right)$.

Note that for flows with attached boundary layers, such as flows in the linear acoustics 
limit, the condition $\left.(\nabla p, \mathbf{n})\right|_{\partial \Omega_{B}}=0$ is satisfied. In the case of adiabatic wall in order for the normal pressure gradient at the boundary to vanish, from the equation of state it follows that the density should satisfy the Neuman boundary condition $\left.(\nabla \rho, \mathbf{n})\right|_{\partial \Omega_{B}}=0$.

For more general flows, when $\left.(\nabla p, \mathbf{n})\right|_{\partial \Omega_{B}} \neq 0$, the passive evolutionary condition on density could be enforced through the CBVP method. In this case the inhomogeneous boundary condition on the normal derivative of the density at the surface $\left.(\nabla \rho, \mathbf{n})\right|_{\partial \Omega_{B}}=\Phi(\mathbf{x})$ could be imposed as

$$
\frac{\partial \rho}{\partial t}=(1-\chi) R H S_{\rho}-\underbrace{\frac{\chi}{\eta_{c}}\left(n_{k} \frac{\partial \rho}{\partial x_{k}}-\Phi(\mathbf{x}, t)\right)}_{\text {penaly term }},
$$

where $\Phi(\mathbf{x}, t)$ is the solution of initial boundary value problem for the transport equation

$$
\frac{\partial \Phi}{\partial t}=-\frac{\chi}{\eta_{c}} \frac{\partial \Phi}{\partial n},\left.\quad \Phi\right|_{\partial \Omega_{B}}=\left.\frac{\partial \rho}{\partial n}\right|_{\partial \Omega_{B}} .
$$
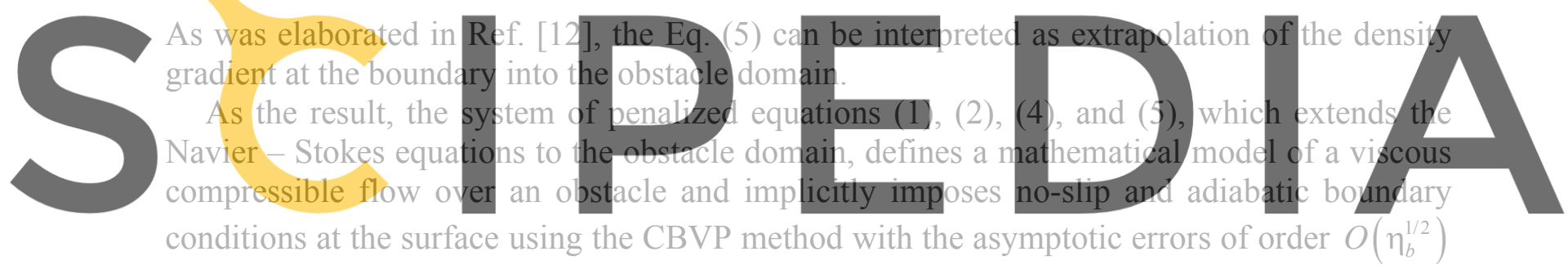

conditions at the surface using the CBVP method with the asymptotic errors of order $O\left(\eta_{b}^{1 / 2}\right)$

the Eq. (3)).

\section{COMPUTATIONAL APPROACH}

The system of equations (1), (2), (4) in conservative form is solved together with Eq. (5) by numerical integration in the whole computational domain $\Omega$ containing the obstacle $\Omega_{B}$. For the spatial integration hybrid finite-volume method with high order EBR schemes in the flow region and low-order finite-difference approximation inside of the obstacle is used.

The spatial discretization in the domain $\Omega_{E}=\Omega \backslash \bar{\Omega}_{B}$ is based on the vertex-centered formulation, which means that all the unknown variables are determined at mesh nodes surrounded by computation cells (dual mesh). Convective fluxes are approximated using quasione-dimensional reconstruction of the variables along a mesh edge (EBR scheme). The viscous terms in the Navier-Stokes system are approximated using the finite-element method with linear basis functions [13].

The system of equations (1), (2), (4), (5) in the domain $\bar{\Omega}_{B}$ could be represented in the matrix-vector form: 


$$
\frac{\partial \mathbf{Q}}{\partial t}=\mathbf{A} \frac{\partial \mathbf{Q}}{\partial n}+\mathbf{S}
$$

where $\mathbf{Q}=(\rho, \rho u, \rho v, E, \Phi)^{T}-$ vector of conservative variables,

$$
\mathbf{A}=\frac{1}{\eta_{c}}\left(\begin{array}{lllll}
-1 & 0 & 0 & 0 & 0 \\
-u & 0 & 0 & 0 & 0 \\
-v & 0 & 0 & 0 & 0 \\
-\left(u^{2}+v^{2}\right) & u & v & -1 & 0 \\
0 & 0 & 0 & 0 & -1
\end{array}\right), \quad \mathbf{S}=\left(\begin{array}{l}
\frac{1}{\eta_{c}} \Phi \\
-\frac{1}{\eta_{b}} \rho\left(u-u_{w}\right)+\frac{1}{\eta_{c}} u \Phi \\
-\frac{1}{\eta_{b}} \rho\left(v-v_{w}\right)+\frac{1}{\eta_{c}} v \Phi \\
-\frac{1}{\eta_{b}}\left[\rho u\left(u-u_{w}\right)+\rho v\left(v-v_{w}\right)\right]+\frac{1}{\eta_{c}} \frac{E}{\rho} \Phi \\
0
\end{array}\right) .
$$

Without loss of generality, let us consider an unstructured mesh with triangular cells $T_{j}$ covering the domain $\bar{\Omega}_{B}: \bar{\Omega}_{B}=\bigcup T_{j}$ and $T_{j} \cap T_{i}=\varnothing(i \neq j)$.

For the numerical solution of system (6) an implicit finite difference scheme is constructed:
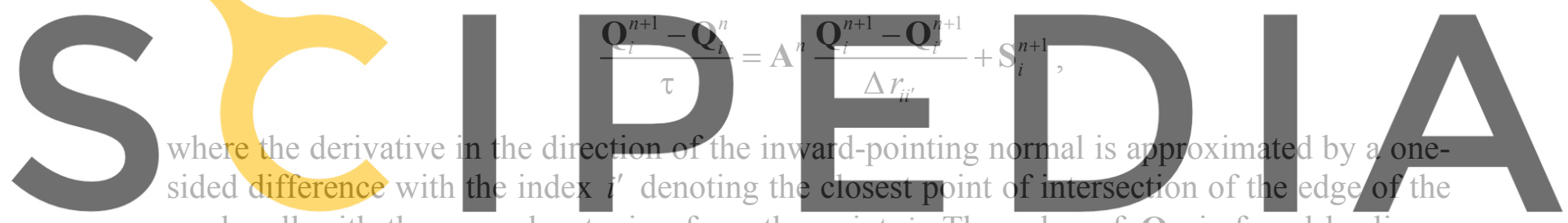

mesh cell with the normal outgoing from the point $i$. The value of $\mathbf{Q}_{i^{\prime}}$ is found by linear

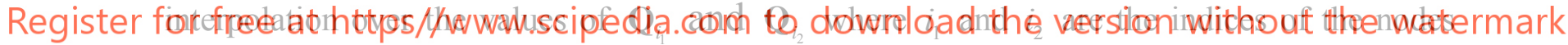

incident to the intersected edge contained point $i^{\prime}$. Note that the scheme introduces an implicit numerical diffusion proportional to the cell size, used to approximate the normal derivatives in system (6). Therefore, the explicit numerical diffusion term $v_{n} \Delta \mathbf{u}$ in Eq. (1) could be omitted.

An implicit second order time integration method is used to solve the system of equations (1), (2), (4) and (5) in the whole computational domain. The solutions in $\Omega_{E}$ and $\bar{\Omega}_{B}$ domains are coupled through interactions defined by the approximation stencil of the normal derivatives in the equations (5) and the variable reconstruction stencil used in the approximation of the conservative form of the Navier-Stokes equations in the fluid region. For each iteration during the implicit integration step the corresponding linearized system of equations is solved using BiCGStab method [14].

\section{NUMERICAL RESULTS}

In this section, the results of numerical simulation of three test problems with exact analytical solutions are discussed. In addition, the results of CBVP simulations are compared with the numerical solutions of the same problems using Brinkman volume penalization method with 
the same time integration and spatial discretization methods [15].

\subsection{Acoustic pulse reflection}

Let us start by considering the problem of acoustic pulse reflection from the solid boundary. The penalized equations are solved in a computational domain $\Omega=[-0.65,0.25] \times[0,0.1]$ with the immersed boundary located at $x=0$ and the penalization region $\bar{\Omega}_{B}=[0,0.25] \times[0,0.1]$.

The numerical computations are conducted on the structured rectangular mesh (SRM3) and a sequence of quasi-isotropic unstructured triangular meshes (UTM1, UTM2, UTM3) with progressively refined mesh element size. For comparison, the numerical results are also obtained for anisotropic triangular mesh (UTM4) with the element size near the wall matching the size of the UTM3 mesh with continuous increase of the element size with the stretching factor of 1.02, so that away from the interface the mesh approaches UTM1 resolution. This problem is solved in the inviscid limit with $\operatorname{Re}=10^{8}$ and the penalization parameters $\eta_{b}=10^{-6}$ and $\eta_{c}=10^{-2}$

The no-slip and adiabatic boundary conditions at the fluid-solid interface are approximated by CBVP method. The periodic boundary conditions are applied in the vertical direction and the Dirichlet boundary conditions for the unperturbed medium are used on the left and right computational boundaries.
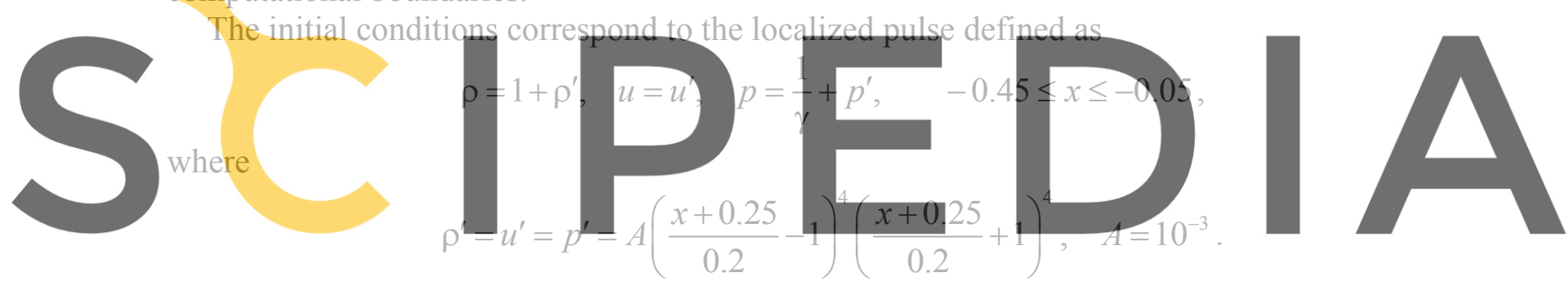

Register for free at https//www.scipedia.com to download the version without the watermark

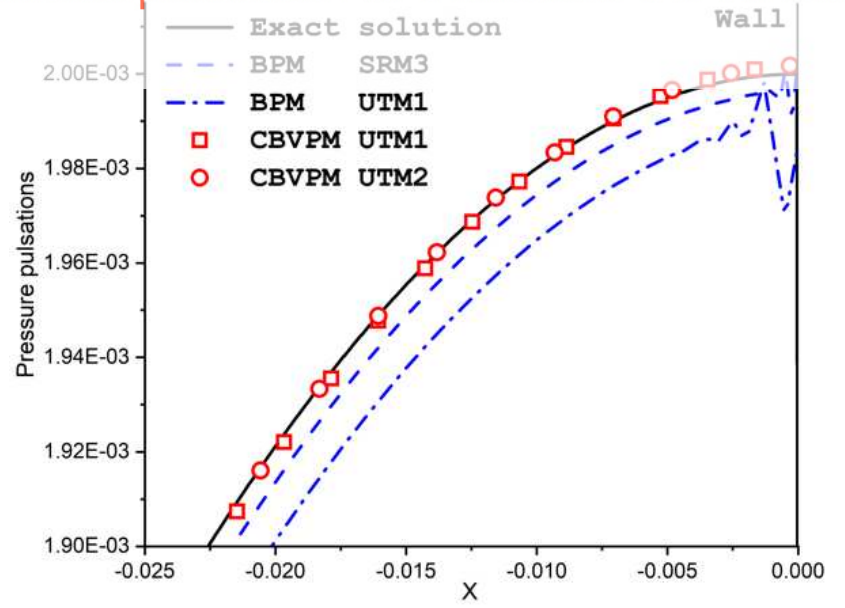

Figure 1: Pressure pulsation of the wave at the time of reflection

The pulse, initially propagating toward the solid wall, reflects and start moving in opposite direction. Figure 1 shows the CBVP and BP solutions for the pressure near the wall at the time 
$t=0.25$ (the time of reflection from the wall) together with the exact solution shown for comparison. A good agreement between the analytical solution and the CBVP solutions even on the coarse mesh is observed. In contrast the error of the BP solution is noticeable even on the finest mesh.

The mesh convergence for CBVP and BP solutions is shown in Table 1. The errors of the numerical solution for the pressure fluctuations in the root-mean-square norm, are calculated in the flow domain at the time $t=0.5$.

Table1: $L_{2}$-norm error of reflected pulse for $t=0.5$.

\begin{tabular}{lccccc}
\hline & SRM3 & UMT1 & UMT2 & UMT3 & UMT4 \\
\hline BPM & $4.72 \mathrm{e}-6$ & $1.33 \mathrm{e}-5$ & $7.33 \mathrm{e}-6$ & $3.71 \mathrm{e}-6$ & $4.93 \mathrm{e}-6$ \\
\hline CBVPM & $9.82 \mathrm{e}-7$ & $3.04 \mathrm{e}-6$ & $1.67 \mathrm{e}-6$ & $8.26 \mathrm{e}-7$ & $7.20 \mathrm{e}-7$ \\
\hline
\end{tabular}

The errors in columns UMT1-UMT3 confirm the linear convergence of the penalized solution with the error approximately decreasing by the factor of 2 when the mesh resolution is refined by the same factor. The results for the anisotropic UMT4 mesh are close to the simulations on the fine UMT3 mesh. Thus, it is confirmed that the error is defined by the type of volume penalization and the mesh size in the vicinity of the wall. The results reported in Table 1 clearly demonstrate that the Brinkman volume penalization method has a lower accuracy than the characteristic-based volume penalization method.
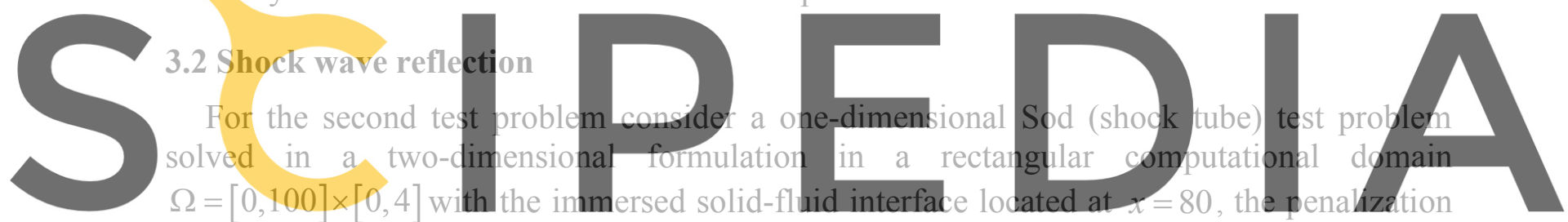

domain $\bar{\Omega}_{B}=[80,100] \times[0,4]$ and the initial shock location at $x=30$. The problem is solved for

Register for free at https///www.scipedia.com to download the version without the watermark three quasi-isotropic triangular unstructured meshes with the following characteristic element sizes: 1 (UMT1 grid), 0.5 (UMT2 grid), 0.25 (UMT3 grid).

The discontinuous initial conditions are given by

$$
(\rho, u, v, p)= \begin{cases}(\rho, u, v, p)_{1}=(1,0,0,1 / \gamma) & x \geq 30 \\ (\rho, u, v, p)_{2}, & x<30\end{cases}
$$

where the flow state in front of the shock $(\rho, u, v, p)_{2}$ is chosen according to the RankineHugoniot jump conditions.

This problem is solved in the inviscid limit with $\mathrm{Re}=10^{7}$ and the penalization parameters $\eta_{b}=10^{-6}$ and $\eta_{c}=10^{-6}$. No-slip and adiabatic boundary conditions are imposed implicitly on the solid wall at $x=80$ through CBVP and BP methods.

The pressure distributions of the reflected shock wave at time $t=60$ for CBVP and BP simulations are presented in Fig. 2, where the exact analytical solution is also shown for the reference. As can be seen from the figure, the numerical solutions obtained by CBVP method on fine (UMT3) and coarse (UMT1) mesh are in good agreement with the analytical solution. 
In contrast the BP solutions on the same meshes clearly underestimate the pressure of the reflected shock wave with the noticeable phase lag, which is mainly due to incorrect definition of the energy flux at the boundary and the pressure seepage, caused by the strong pressure gradient inside of the penalization region. The CBVP simulations result in the correct pressure gradient on the solid wall, due to the correct calculation of the density gradient, extrapolated from the solid boundary, and the temperature gradient imposed thorough adiabatic boundary conditions at the interface of the "immersed" domain, resulting in a zero energy flux at the boundary and, as a consequence, the correct reflection of the shock wave.

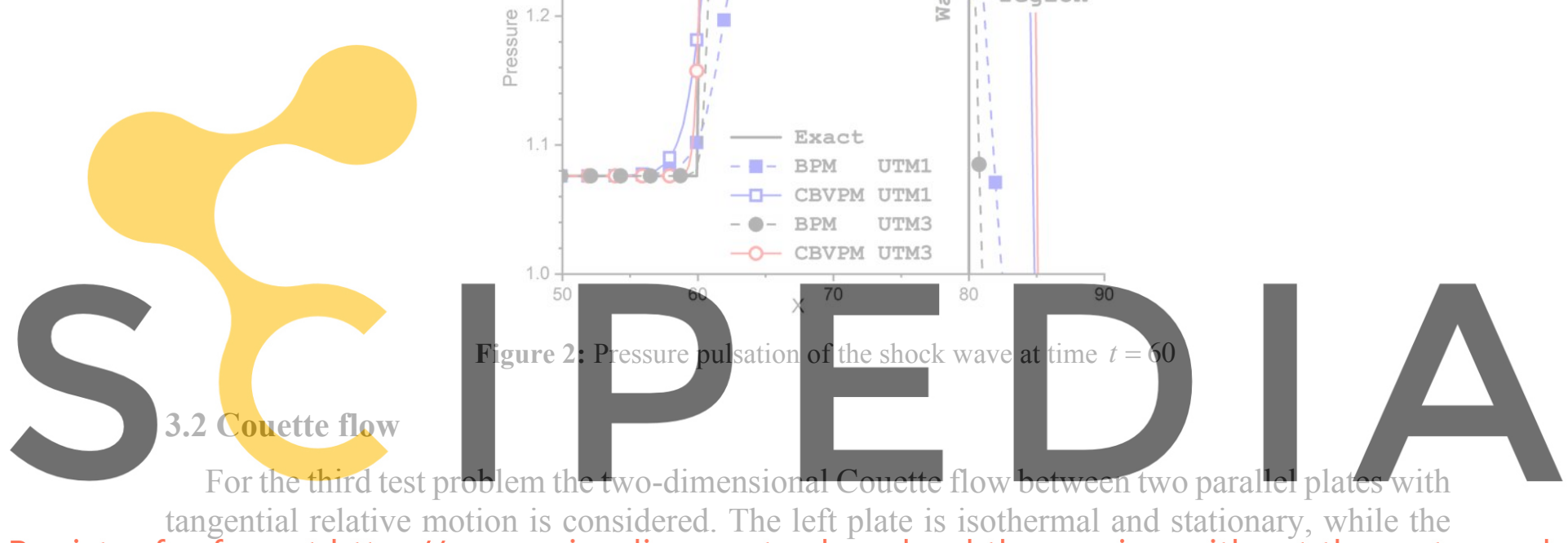

tangential relative motion is considered. The left plate is isothermal and stationary, while the

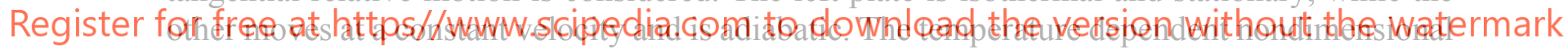

dynamic viscosity of form $\mu=\left(T / T_{0}\right)^{-1 / 2} / \mathrm{Re}$ is assumed, where $T_{0}$ is the plate temperature.

The problem is solved for $\operatorname{Re}=1$, constant Prandtl number $\operatorname{Pr}=1$, and the penalization parameters $\eta_{b}=10^{-3}$ and $\eta_{c}=10^{-2}$.

The penalized equations are solved in the computational domain $\Omega=[-0.5,0.5] \times[0,0.02]$ using quasi-uniform triangular meshes with the following typical mesh cell sizes: $\Delta x=0.02$ (UMT1 mesh), 0.01 (UMT2 mesh), 0.005 (UMT3 mesh).

The plates are located perpendicular to the $O X$ axis with the surfaces located along $x_{L}=-0.5$ and $x_{R}=0.2$ planes. On the left boundary of the computational domain, which coincides with the surface of the left plate $\left(x=x_{L}\right)$, the isothermal $\left(T_{L}=3 / \gamma\right)$ and no-slip boundary conditions are imposed. The constant velocity $v_{P}=10$ and adiabatic $\partial T / \partial x=0$ boundary conditions on the right plate $\left(x=x_{R}\right)$ are imposed implicitly using the characteristic based volume penalization method. The simulations using Brinkman volume penalization method are also caried out for comparison. 
The problem has the exact solution [16] which can be written as

$$
\begin{gathered}
T(x)=\tau^{2}(x), \\
\tau(x)=\sqrt{T_{L}} \frac{\cos \left(c\left(x_{R}-x\right)\right)}{\cos \left(c\left(x_{R}-x_{L}\right)\right)}, \quad c=\frac{1}{\left(x_{R}-x_{L}\right)} \operatorname{arctg}\left(\frac{v_{R}}{\sqrt{T_{L}}}\left(\frac{\operatorname{Pr}(\gamma-1)}{2 \gamma}\right)^{1 / 2}\right), \\
v(x)=v_{R}-\left(\frac{\operatorname{Pr}(\gamma-1)}{2 \gamma}\right)^{-1 / 2} \sqrt{T_{L}} \frac{\sin \left(c\left(x_{R}-x\right)\right)}{\cos \left(c\left(x_{R}-x_{L}\right)\right)} .
\end{gathered}
$$

The numerical simulations are continued until a stationary solution independent of $y$ coordinate is obtained. The velocity and temperature profiles near the right boundary, computed on the coarse (UMT1) and fine (UMT3) meshes using CBVP and BP methods are shown in Fig. 3, where exact solution is also shown for comparison. The numerical simulations using the Characteristic-Based Volume Penalization method result in more accurate solutions compared to the Brinkman volume penalization method.
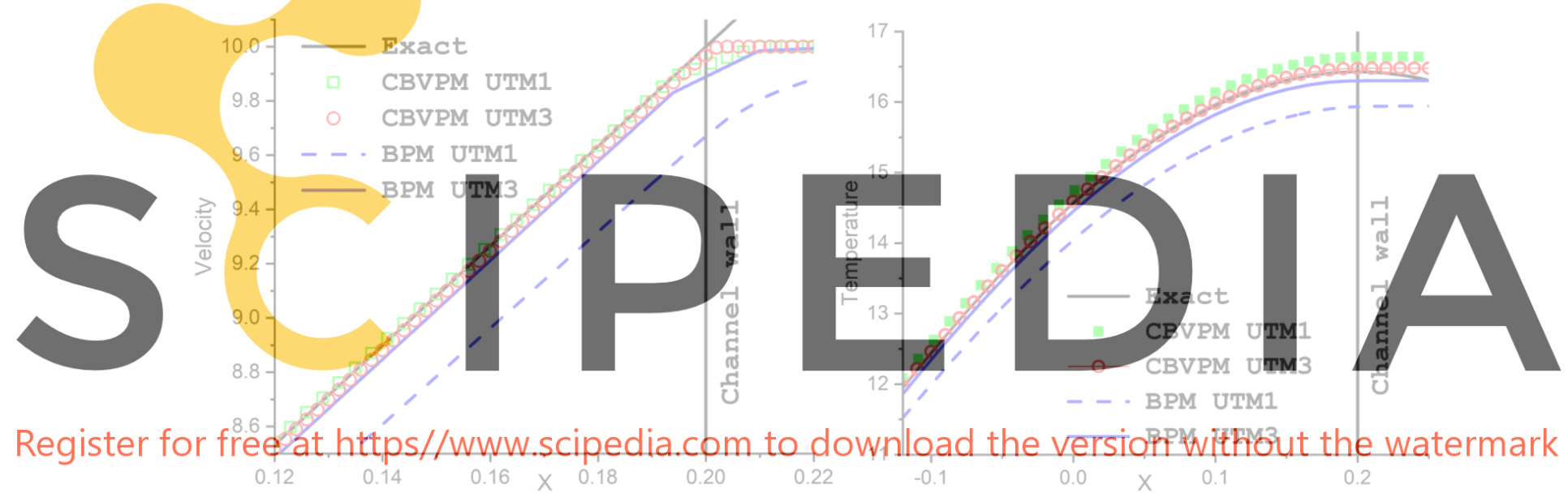

Figure 3: Velocity and temperature near the right plate for Couette flow problem

Table2: $L_{2}$-error of the CBVP solution for the temperature

\begin{tabular}{ccc}
\hline UMT1 & UMT2 & UMT3 \\
\hline $1.07 \mathrm{e}-1$ & $4.05 \mathrm{e}-2$ & $1.83 \mathrm{e}-2$ \\
\hline
\end{tabular}

Table 2 shows the mesh convergence for CBVP method. The root-mean-square errors for numerical solution calculated over flow domain $\Omega \backslash \Omega_{B}$ for different meshes demonstrate the linear convergence of CBVP method caused by the dominance of the numerical error over the penalization error introduced by CBVP method for the given choice of the penalization parameters that are held constant [9]. Note that, in contrast to the previous test problems, in this problem, an inhomogeneous no-slip boundary conditions are modeled. 


\section{CONCLUSIONS}

A method for numerical simulation of compressible flows over solid obstacles using nonboundary conforming unstructured meshes is presented. The mathematical model is based on the Navier-Stokes equations and makes use of the characteristic-based volume penalization method to mimic the effect of the obstacle on a flow. The CBVP method allows to impose general (Dirichlet, Neumann and Robin) boundary conditions and is relatively simple to implement numerically. The penalized Navier-Stokes equations are solved to simulate compressible flow around obstacles. In the solid region the hyperbolic penalization equations are solved to propagate the solution from the surface along the inward-pointing characteristics that enforce the desired value of derivative with an a priori defined accuracy. The accuracy and efficiency of the developed method is demonstrated for three cases with the increasing complexity: the acoustic pulse and shock wave reflection problems and the Couette flow. The results for all three cases are in good agreement with the analytical solutions. The CBVP methods demonstrate consistently more accurate solutions compared to the numerical solution of the same problem obtained using Brinkman volume penalization method. The CBVP method maintains rigorous control of the error through a priori chosen parameters for all boundary conditions.

\section{REFERENCES}

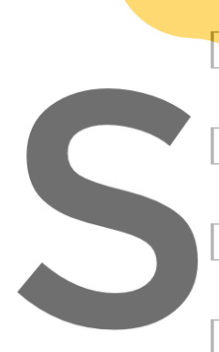

Angot, Ph., Bruneau, C.-H. and Fabrie, P. A penalization method to take into account obstacles in incompressible viscous flow
Carbou, G. and Fabrie, P. Borndary
incompressible flow. Adv. Differ Equ. (200
Bergmann, M. and Iollo, A. Modeling an
Phys. (2011) 230:329-34.8. Kevlahan, N. and Ghidaglia, J.-M
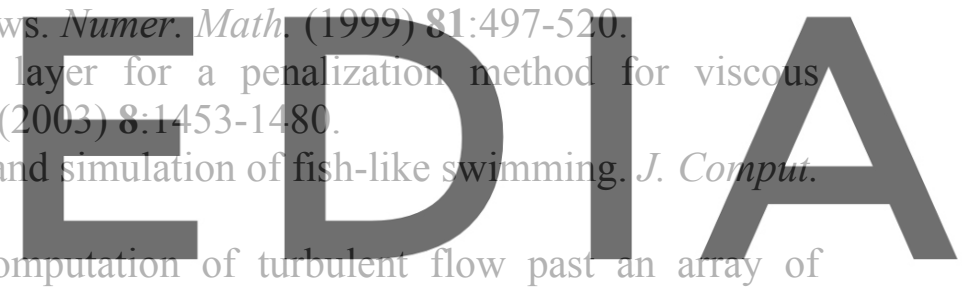
cylinders using a spectral method with Brinkman penalization. Eur. J. of Mech.- B/Fluids,

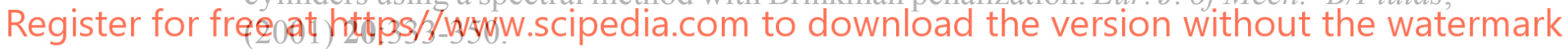

[5] Vasilyev, O. and Kevlahan, N. Hybrid wavelet collocation-Brinkman penalization method for complex geometry flows. Int. J. Num. Meth. Fluids (2002) 40:531-538.

[6] Spietz, H.J., Hejlesen, M.M. and Walther, J.H. Iterative Brinkman penalization for simulation of impulsively started flow past a sphere and a circular disc. J. Comput. Phys., (2017) 336:261-274.

[7] Liu, Q. and Vasilyev, O.V. Brinkman Penalization method for compressible flows in complex geometries. J. Comput. Phys. (2007) 227:946-966.

[8] Komatsu, R., Iwakami, W. and Hattori, Y. Direct numerical simulation of aeroacoustic sound by volume penalization method. Comput. Fluids (2016), 130:24-36.

[9] Brown-Dymkoski, E., Kasimov, N. and Vasilyev, O.V. A characteristic based volume penalization method for general evolution problems applied to compressible viscous flows. J. Comput. Phys. (2014) 262:344-357.

[10] Nejadmalayeri, A., Vezolainen, A., Brown-Dymkoski, E. and Vasilyev, O.V. Parallel Adaptive Wavelet Collocation Method for PDEs. J. Comput. Phys. (2015) 298:237-253.

[11] Bakhvalov, P., Abalakin, I. and Kozubskaya, T. Edge-based reconstruction schemes for unstructured tetrahedral meshes. Int. J. Numer. Meth. Fluids (2016) 81:331-356. 
[12] Aslam, T.D. A partial differential equation approach to multidimensional extrapolation. J. Comput. Phys. (2003) 193:349-355.

[13] Koobus, B., Alauzet, F. and Dervieux, A. Numerical algorithms for unstructured meshes. In F. Magoulès (Ed.) Computational Fluid Dynamics (pp. 131-203) Boca Raton: Chapman \& Hall/CRC, 2011.

[14] van der Vorst, H.A. BICGSTAB: A fast and smoothly converging variant of BICG for the solution of nonsymmetric linear systems. SIAM J. Sci. Comput. (1992) 13:631-644.

[15] Abalakin, I.V., Gorobets, A.V., Zhdanova, N.S. and Kozubskaya, T.K. Using Brinkman penalization method for numerical simulation viscous compressible flows over obstacles. Preprint KIAM 11 (2014) (in Russian).

[16] ColESo (Collection of Exact Solutions): available at: https://github.com/bahvalo/ColESo. 